\title{
Anesthetic management of the first forearm transplantation in Korea
}

\author{
Jinyeol Kwon, Sung Mee Jung, Sae-Yeon Kim, Nyeong Keon Kwon, and \\ Sang-Jin Park \\ Department of Anesthesiology and Pain Medicine, Yeungnam University College of Medicine, Daegu, Korea
}

Vascularized composite allotransplantation for the forearm is a complex surgical procedure, requiring multidisciplinary collaboration. It is important to provide optimal blood flow to the grafts, effective immunosuppression, and early rehabilitation for graft survival and good functional outcomes. As ischemia-reperfusion injury and substantial but unquantifiable blood loss are inevitable in this type of surgery, anesthetic management should focus on providing adequate hemodynamic management with proper monitoring, and anesthetic and analgesic strategies to prevent vasoconstriction in the graft. In this paper, we describe the anesthetic management of the first forearm transplantation performed in Korea.

Keywords: Anesthesia; Fluid therapy; Forearm; Graft survival; Vascularized composite allotransplantation.

Upper-extremity transplantation is not a life-saving procedure, but it has been found to result in a marked improvement in the aesthetic and functional qualities of life [1]. It is a complex procedure, involving vascularized composite allotransplantation (VCA) of different tissues including muscles, tendons, bones, blood vessels, nerves, and skin, and requires multidisciplinary collaboration among surgeons, anesthesiologists, immunologists, psychologists, and rehabilitators. Since the first successful hand transplantation in 1998 in France, improved graft survival-owing to advances in immunosuppressive therapy and microsurgical techniques-has resulted in more than 107 cases of upper-limb transplantation worldwide till 2015 [1]. The first guideline for the anesthetic management of upper-extremity transplantation, the Pittsburgh Upper Extremity Transplant An-

Corresponding author: Sung Mee Jung, M.D., Ph.D.

Department of Anesthesiology and Pain Medicine, Yeungnam University College of Medicine, 170, Hyeonchung-ro, Nam-gu, Daegu 42415, Korea

Tel: 82-53-620-3368, Fax: 82-53-626-5275

Email: applejsm@gmail.com

ORCID: https://orcid.org/0000-0001-5602-9011

Received: July 19, 2017.

Revised: August 25, 2017 (1st); September 20, 2017 (2nd).

Accepted: September 25, 2017.

Korean J Anesthesiol 2018 February 71(1): 66-70

https://doi.org/10.4097/kjae.2018.71.1.66 esthesiology Protocol (PUETAP), was based on a single center study with eight upper extremity transplants [2]. Further studies are required to ensure standardization of anesthetic protocols across individual transplant centers. In this paper, we describe the anesthetic management of the first forearm transplantation performed in Korea.

\section{Case Report}

A 35-year-old man $(66 \mathrm{~kg}, 182 \mathrm{~cm})$ was selected for forearm VCA. He had a medical history of left forearm amputation due to trauma 18 months prior to the consultation (Fig. 1). At the time of the forearm transplantation, he had no coexisting medical conditions or psychological issues. A 49-year-old brain-dead male donor was selected on the basis of blood typing and human leukocyte antigen typing.

Before the surgery, the patient had fasted for eight hours; no premedication was administered. Noninvasive blood pressure, electrocardiogram, and oxygen saturation $\left(\mathrm{SpO}_{2}\right)$ monitoring were initiated. Anesthesia was induced with propofol (2 mg/ $\mathrm{kg}$ ) and a continuous infusion of remifentanil $(1 \mu \mathrm{g} / \mathrm{kg} / \mathrm{h})$. Orotracheal intubation was performed after administration of rocuronium $(0.75 \mathrm{mg} / \mathrm{kg})$. Anesthesia was maintained with sevoflurane with $50 \%$ oxygen to maintain the bispectral index range between 40 and 60 . Remifentanil and rocuronium were continuously administered to provide adequate analgesia and

(c) This is an open-access article distributed under the terms of the Creative Commons Attribution Non-Commercial License (http://creativecommons.org/ licenses/by-nc/4.0/), which permits unrestricted non-commercial use, distribution, and reproduction in any medium, provided the original work is properly cited. 

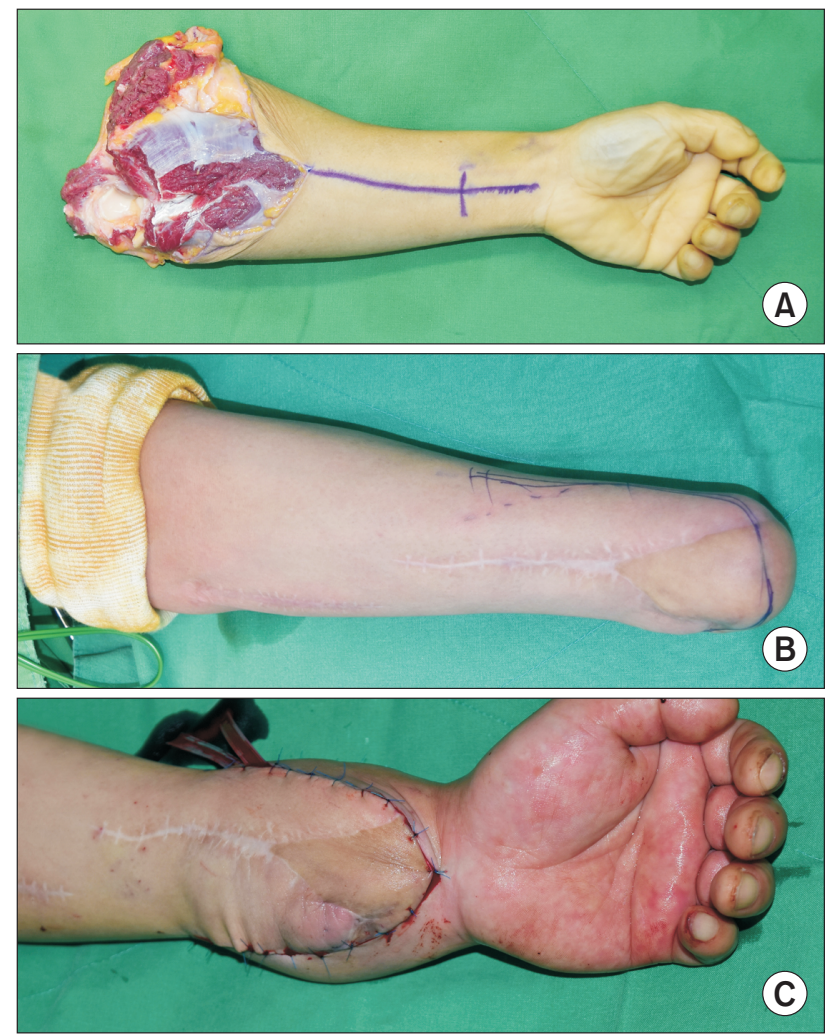

Fig. 1. Intraoperative appearance of the forearm. (A) Donor's forearm after harvest. (B) Recipient's forearm before preparation. (C) Transplanted forearm after surgery.

muscular relaxation, respectively. A $20-\mathrm{G}$ vascular catheter was inserted into the right radial artery for invasive monitoring of arterial pressure, arterial blood gas analysis (ABGA), and laboratory testing during surgery. A triple-lumen vascular catheter was introduced into the right subclavian vein to monitor central venous pressure (CVP) and administer fluids and medication. A 16-G vascular catheter was also placed in a superficial vein in the left leg. A warm blanket with a heated circuit, fluid warmer, and forced-air warmer were used to maintain normothermia during surgery.

Intravenous ceftriaxone $(2 \mathrm{~g})$, acyclovir $(250 \mathrm{mg})$, and fluconazole $(500 \mathrm{mg})$ were administered preoperatively for antimicrobial prophylaxis. Immunosuppression was induced with basiliximab (IL-2 receptor blocker, $20 \mathrm{mg}$ ) and maintained with triple-drug therapy using methylprednisolone (125 mg), mycophenolate mofetil (an inhibitor of inosine monophosphate dehydrogenase, $750 \mathrm{mg}$ ), and tacrolimus (a calcineurin inhibitor, 5 $\mathrm{mg})$.

Preparation of the donor's forearm and dissection of the recipient's forearm were performed simultaneously. The left forearm of the donor was procured at the proximal humeral level before aortic cross-clamping and immediately perfused

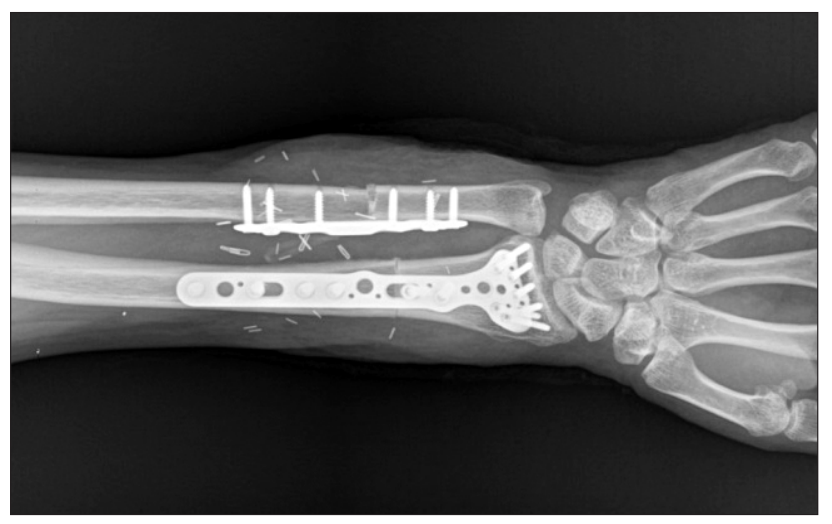

Fig. 2. Bony fixation of the forearm at the distal portion of the radius and ulna.

with a cold histidine-tryptophan-ketoglutarate solution before transplantation (Fig. 1). The recipient's forearm was dissected under a pneumatic tourniquet inflated to $300 \mathrm{mmHg}$ with intermittent deflation over 125 minutes. The forearm preparation was followed by bony fixation with a plate at the distal radius and ulna, followed by end-to-end anastomoses of the ulnar and anterior interosseous arteries and four venae comitantes (Fig. 2). Intravenous heparin (5000 units) was administered just before the forearm was reperfused.

The patient's esophageal temperature was maintained at $36-37^{\circ} \mathrm{C}$. The transplanted hand turned pinkish immediately after arterial anastomosis and reperfusion. The ischemic-time from the retrieval of the donor's forearm to reperfusion following transplantation was 210 minutes. Although the patient continued to bleed from the surgical site during the vascular anastomoses, the mean arterial pressure (MAP) and heart rate were maintained at $65-85 \mathrm{mmHg}$ and $80-100$ beats/min, respectively, by administration of fluid and packed red blood cells (pRBCs, Table 1). Low-dose dopamine (3-5 $\mu \mathrm{g} / \mathrm{kg} / \mathrm{min})$ was infused to maintain MAP above $65 \mathrm{mmHg}$ during the reconstruction of the tendons and the ulnar, median, and radial nerves. Skin grafting for monitoring acute rejection was then performed. On completion of the surgery, the patient underwent ultrasound-guided axillary brachial plexus block (BPB) with ropivacaine $(0.375 \%$, $20 \mathrm{ml}$ ) to provide postoperative analgesia and adequate blood flow to the allograft. Immediately after surgery, the transplanted hand was slightly edematous but pinkish (Fig. 1). $\mathrm{SpO}_{2}$ values measured on the right and left second fingers were $100 \%$ and $98 \%$, respectively.

The duration of surgery and anesthesia was 8 hours $10 \mathrm{~min}$ utes and 9 hours 10 minutes, respectively. The estimated blood loss was $1,500 \mathrm{ml}$, and urine output was $2,230 \mathrm{ml}$. The patient received 11,100 ml (168 ml/kg) of crystalloid, 1,000 ml (15 ml/ $\mathrm{kg}$ ) of colloid, and 4 units of pRBCs. Because excessive bleeding or coagulopathy was not observed during surgery, hemostatic 
Table 1. Changes in Hemodynamic and Laboratory Data

\begin{tabular}{|c|c|c|c|c|c|c|c|}
\hline & \multirow{2}{*}{$\begin{array}{c}\text { After } \\
\text { anesthesia }\end{array}$} & \multirow{2}{*}{$\begin{array}{l}30 \text { min before } \\
\text { reperfusion }\end{array}$} & \multicolumn{4}{|c|}{ After reperfusion } & \multirow{2}{*}{$\begin{array}{l}\text { End of } \\
\text { surgery }\end{array}$} \\
\hline & & & $5 \mathrm{~min}$ & $1 \mathrm{~h}$ & $2 \mathrm{~h}$ & $3 \mathrm{~h}$ & \\
\hline $\mathrm{ABP}(\mathrm{mmHg})$ & $120 / 65$ & $115 / 60$ & $100 / 50$ & $99 / 50$ & $110 / 55$ & $120 / 65$ & $130 / 71$ \\
\hline Heart rate (bpm) & 85 & 105 & 100 & 95 & 81 & 87 & 90 \\
\hline CVP (mmHg) & 7 & 9 & 10 & 12 & 13 & 12 & 13 \\
\hline $\mathrm{pH}$ & 7.46 & 7.45 & 7.46 & 7.46 & 7.45 & 7.47 & 7.46 \\
\hline $\mathrm{PaCO}_{2}(\mathrm{mmHg})$ & 36 & 37 & 36 & 35 & 37 & 35 & 37 \\
\hline $\mathrm{PaO}_{2}(\mathrm{mmHg})$ & 234 & 234 & 227 & 200 & 221 & 228 & 210 \\
\hline $\mathrm{HCO}_{3}^{-}(\mathrm{mmol} / \mathrm{L})$ & 25.6 & 25.7 & 25.6 & 24.9 & 25.7 & 25.5 & 26.3 \\
\hline $\mathrm{Hb}(\mathrm{g} / \mathrm{dl})$ & 15.0 & 12.2 & 11.6 & 11.2 & 10.5 & 9.9 & 10.9 \\
\hline $\mathrm{Ca}^{2+}(\mathrm{mmol} / \mathrm{L})$ & 3.49 & 3.85 & 3.81 & 3.21 & 3.77 & 3.61 & 3.29 \\
\hline $\mathrm{K}^{+}(\mathrm{mmol} / \mathrm{L})$ & 3.7 & 3.9 & 4.1 & 4.0 & 4.1 & 3.9 & 3.8 \\
\hline Glucose (mg/dl) & 147 & 143 & 152 & 137 & 150 & 165 & 184 \\
\hline Lactate $(\mathrm{mmol} / \mathrm{L})$ & 1.9 & 3.0 & 2.9 & 3.3 & 3.4 & 2.2 & 2.2 \\
\hline
\end{tabular}

ABP: arterial blood pressure, CVP: central venous pressure.

management guided by a thromboelastogram and coagulation panels including a prothrombin time, partial thromboplastin time, international normalized ratio, and fibrinogen level was not performed. The patient was extubated with sugammadex (3 $\mathrm{mg} / \mathrm{kg}$ ) after the return of consciousness and adequate spontaneous ventilation.

The forearm and hand remained pinkish and slightly edematous in the immediate postoperative period. Adequate blood flow to the transplanted forearm was confirmed by Doppler ultrasound. $\mathrm{SpO}_{2}$ measured at the left second finger was maintained in a range of $98-100 \%$. The visual analog scale was 3 in the immediate postoperative period and maintained between 2 and 3 after receiving intravenous meperidine $(12.5 \mathrm{mg})$ on the first postoperative day.

Two episodes of acute rejection occurred at days 6 and 47 post-transplantation. In both episodes, rejection was suspected due to erythematous changes on the transplanted hand. The graft-skin biopsy confirmed mild acute rejection (Grade I: mild infiltration of lymphocytes in the dermis) [3]. The patient received steroid pulse therapy and anti-thymocyte globulin, according to the immunosuppression protocol; tacrolimus ointment was applied to the areas of skin showing signs of rejection. The patient was discharged from hospital 22 days postoperatively. Once graft viability was confirmed, an early rehabilitation protocol was initiated. The patient had recovered his pain and tactile sensation and could grasp a baseball with his fingers at first discharge. His second admission due to acute rejection also resolved with tacrolimus ointment, increased in immunosuppressive dosage, and plasma exchange.

\section{Discussion}

This case report describes the anesthetic management of the first forearm-transplantation patient in Korea. Contrary to solid tissue transplantation, forearm transplantation is classified as VCA, involving muscle, tendon, nerve, blood vessel, bone, and skin. To improve graft survival and minimize the risk of perioperative morbidity and mortality, we focused on maintaining optimal blood flow to the allograft.

The goal of preoperative evaluation in a patient scheduled for upper-extremity transplantation is to assess and plan to avoid comorbidity-associated risk and optimize blood flow to the VCA. The anesthesiologists should determine how to coordinate vascular access and intraoperative monitoring with patient positioning during the procedure.

Anesthetic management in patients undergoing VCA is similar to that for a solid organ transplant. However, replantation microsurgery to ensure optimal blood flow to the graft, maintaining homeostasis to improve graft survival and protect against ischemia-reperfusion injury (IRI), and significant blood loss during the procedure should be considered.

Ischemia-reperfusion injury is an inevitable consequence of donor forearm retrieval owing to the temporary deprivation of blood supply before being reperfused following transplantation. It is critical to minimize ischemic time, which is closely related to the severity of IRI. The donor limb should be procured before other solid organ retrieval and cross-clamping of the aorta. Reperfusion of the ischemic limb with arterial anastomoses induces endothelial dysfunction and an inflammatory response characterized by reactive vasodilation, sequestration of leukocytes and platelets, increased microvascular permeability, and generation of oxygen-free radicals and inflammatory mediators [4,5]. It also elicits hemodynamic and metabolic changes such as hypotension, lactic acidosis, and hyperkalemia. The amount of IRI and blood loss during the procedure is also dependent on the anatomical level of transplantation. We adequately managed 
the hemodynamic and metabolic changes due to IRI in this unilateral forearm transplantation by maintaining normovolemia guided by the arterial pressure and CVP, and laboratory tests including ABGA, glucose, lactate, and electrolytes.

Fluid management during VCA is challenging, as it is difficult to quantify blood loss accurately due to continuous diffuse bleeding, use of a large amount of irrigation fluid, and extensive reperfusion during the procedure. In addition, excessive fluid administration results in interstitial tissue edema, which compromises graft perfusion. The greatest hemodynamic change due to blood loss usually occurs after tourniquet deflation, following arterial anastomoses and continues until completion of the venous anastomoses.

Maintaining adequate perfusion pressure with normovolemic hemodilution is essential to provide optimal intravascular volume to the vascularized graft and improve tissue oxygenation by reducing blood viscosity. We aimed to maintain MAP $>65$ $\mathrm{mmHg}$ and a hematocrit of approximately $30 \%$. To achieve adequate intravascular volume without interstitial edema, we compensated for the extracellular fluid loss with balanced isotonic crystalloid and the loss of blood volume with balanced iso-oncotic colloid and pRBCs. When hypotension was unresponsive to fluid and blood administration, low-dose dopamine was used to maintain cardiac output by increasing cardiac contractility through $\beta$-adrenergic receptors and renal perfusion through dopaminergic receptors. Conversely, $\alpha$-adrenergic agonists are not recommended because of an increased risk of vasoconstriction, complicating microvascular surgery. Continuous monitoring of arterial pressure and CVP and serial measurement of ABGA, hemoglobin, and urine output helped guide fluid and blood transfusion management. It is necessary to determine whether goal-directed management of circulating volume using dynamic surrogate parameters such as stroke volume variation and cardiac output may contribute to better outcomes in VCA.

Hypothermia must be avoided because it causes peripheral vasoconstriction and increases blood viscosity. The temperature was maintained at $>36^{\circ} \mathrm{C}$ with a warm blanket, forced air warmer, fluid warmer, heated and humidified anesthetic circuit, and an operating room temperature around $24^{\circ} \mathrm{C}$ throughout the procedure.

No randomized controlled study has demonstrated the superiority of one anesthetic technique over another in patients undergoing VCA. The advantages of regional anesthesia in terms of improving graft perfusion, preventing vasospasm, and reducing thrombosis were emphasized in the PUETAP [2]. However, general anesthesia combined with adequate analgesia is preferred in limb transplantation surgery because of the long duration of the operation and the need to maintain hemodynamic stability and patient immobility. Balanced anesthesia using volatile anesthetics is preferred over total intravenous anesthesia with propofol in VCA [6,7]. Sevoflurane, which was used in this case, is known to attenuate IRI by preserving post-occlusive blood flow in the human forearm [8]. The decreased capillary filtration coefficient of sevoflurane also leads to less interstitial edema during surgery [9]. Remifentanil provides rapid control of hemodynamic changes and continuous analgesia during surgery.

Regional block prevents vasospasm of the graft with its sympatholytic effect and enables early rehabilitation with effective analgesia postoperatively but may lead to vasodilation-associated hemorrhage and hypotension during surgery [10]. Continuous BPB through an indwelling catheter, unilaterally or bilaterally, was successfully used to provide adequate analgesia until the postoperative period in upper extremity transplantation patients $[2,6,11]$. The PUETAP recommended supraclavicular BPB to diminish stress response to tourniquet inflation and provide rapid and long-lasting analgesia with low dose local anesthetics [2]. Recently infraclavicular BPB has become popular, with the advantages of a reduced risk of phrenic nerve palsy, pneumothorax, and catheter dislodgement than supraclavicular BPB, especially in bilateral forearm transplantation $[6,11]$. However, although the previous reports recommended continuous regional block after surgery for effective analgesia and improved graft perfusion, there is no literature addressing the beneficial effect of continuous BPB on graft survival in upper extremity transplantation or replantation patients $[10,12]$. In this case, we performed a single-shot axillary BPB with long-acting local anesthetic followed by intravenous opioid analgesia for postoperative pain management to minimize the risk of infection.

Despite an overall graft survival rate of $83 \%$ worldwide, graft loss after upper extremity transplantation does occur, mainly due to noncompliance and/or insufficient immunosuppression [1]. The VCA was an immunological challenge because it was composed of various tissue components with distinct immunogenicity. Immunosuppressive therapy was initiated before receiving the allograft and maintained indefinitely. Initial treatment with an IL-2 receptor monoclonal antibody targeting recipient $\mathrm{T}$ cells was followed by triple-drug maintenance therapy in this patient. Contrary to solid organ transplantation, VCA has a unique advantage in immunosuppressive management; graft rejection can be recognized by visual inspection and diagnosed by skin biopsy [13]. The episodes of acute rejection were completely reversed owing to early diagnosis and treatment with increased immunosuppressive dose and the application of topical immunosuppressive ointment. High costs and side effects such as infection, diabetes, hypertension, renal insufficiency, hyperlipidemia, and malignancy may reduce compliance to life-long immunosuppressive therapy in patients undergoing VCA [14].

After forearm transplantation, hand motor function is entirely dependent on re-innervation and reactivation of the grafted forearm muscles. Starting with a passive range of motion 
exercise within 24-48 hours, continuous rehabilitation including physical, psychosocial, vocational, and recreational activities is essential to improve graft function. In general, the functional outcome after upper extremity transplantation is dependent on the anatomical level of transplantation. Patients with distal forearm transplants can expect the best results, with limited intrinsic recovery, some discriminative sensation, faster functional recovery, and more specific sensory/motor recovery [1]. Our patient regained pain and tactile sensation and grasping power within a month after surgery.

In conclusion, we have reported on the anesthetic management of the first forearm transplantation performed in Korea, with emphasis on achieving optimal blood flow to the graft. For an optimal surgical outcome, proper patient selection, vigilant monitoring and adequate management of circulating volume changes and IRI, choice of anesthetic technique and regimen to maintain circulation, ventilation, oxygenation, and temperature; adequate immunosuppression; and early rehabilitation with adequate postoperative analgesia should be achieved through detailed preoperative planning and multidisciplinary communication throughout the perioperative period.

\section{ORCID}

Jinyeol Kwon, http://orcid.org/0000-0002-6551-9474

Sung Mee Jung, http://orcid.org/0000-0001-5602-9011

Sae-Yeon Kim, http://orcid.org/0000-0002-7329-2791

Nyeong Keon Kwon, http://orcid.org/0000-0003-0493-2203

Sang-Jin Park, http://orcid.org/0000-0002-4838-2664

\section{References}

1. Shores JT, Brandacher G, Lee WP. Hand and upper extremity transplantation: an update of outcomes in the worldwide experience. Plast Reconstr Surg 2015; 135: 351e-60e.

2. Lang RS, Gorantla VS, Esper S, Montoya M, Losee JE, Hilmi IA, et al. Anesthetic management in upper extremity transplantation: the Pittsburgh experience. Anesth Analg 2012; 115: 678-88.

3. Cendales LC, Kanitakis J, Schneeberger S, Burns C, Ruiz P, Landin L, et al. The Banff 2007 working classification of skin-containing composite tissue allograft pathology. Am J Transplant 2008; 8: 1396-400.

4. Estebe JP, Davies JM, Richebe P. The pneumatic tourniquet: mechanical, ischaemia-reperfusion and systemic effects. Eur J Anaesthesiol 2011; 28: 404-11.

5. Hughes SF, Cotter MJ, Evans SA, Jones KP, Adams RA. Role of leucocytes in damage to the vascular endothelium during ischaemiareperfusion injury. Br J Biomed Sci 2006; 63: 166-70.

6. Clerc M, Prothet J, Rimmelé T. Perioperative management of a bilateral forearm allograft. Hand Surg Rehabil 2016; 35: 215-9.

7. Gurnaney HG, Fiadjoe JE, Levin LS, Chang B, Delvalle H, Gálvez J, et al. Anesthetic management of the first pediatric bilateral hand transplant. Can J Anaesth 2016; 63: 731-6.

8. Lucchinetti E, Ambrosio S, Aguirre J, Herrmann P, Härter L, Keel M, et al. Sevoflurane inhalation at sedative concentrations provides endothelial protection against ischemia-reperfusion injury in humans. Anesthesiology 2007; 106: 262-8.

9. Bruegger D, Bauer A, Finsterer U, Bernasconi P, Kreimeier U, Christ F. Microvascular changes during anesthesia: sevoflurane compared with propofol. Acta Anaesthesiol Scand 2002; 46: 481-7.

10. Su HH, Lui PW, Yu CL, Liew CS, Lin CH, Lin YT, et al. The effects of continuous axillary brachial plexus block with ropivacaine infusion on skin temperature and survival of crushed fingers after microsurgical replantation. Chang Gung Med J 2005; 28: 567-74.

11. Elkassabany NM, Fosnot CD, Shaked A, Kanchwala SK, Kovach SJ 3rd, Chang B, et al. Perioperative management of a patient scheduled for bilateral hand transplant. J Clin Anesth 2013; 25: 224-7.

12. Niazi AU, El-Beheiry H, Ramlogan R, Graham B, von Schroeder HP, Tumber PS. Continuous infraclavicular brachial plexus blockade: effect on survival of replanted digits. Hand Surg 2013; 18: 325-30.

13. Murray JE. Organ transplantation (skin, kidney, heart) and the plastic surgeon. Plast Reconstr Surg 1971; 47: 425-31.

14. Hautz T, Brandacher G, Zelger B, Gorantla VS, Lee AW, Pratschke J, et al. Immunologic aspects and rejection in solid organ versus reconstructive transplantation. Transplant Proc 2010; 42: 3347-53. 